\title{
A MODIFIED HISTOGRAM BASED FAST ENHANCEMENT ALGORITHM
}

\author{
Amany A. Kandeel ${ }^{1}$, Alaa M. Abbas ${ }^{2}$, Mohiy M. Hadhoud ${ }^{3}$, Zeiad El- \\ Saghir $^{1}$ \\ ${ }^{1}$ Dept. of Computer Science and Engineering, \\ Faculty of Electronic Engineering, \\ Univ. of Menoufia, 32952, Menouf, Egypt. \\ amany_1980@hotmail.com, zeiad40@yahoo.com \\ ${ }^{2}$ Dept. of Electronics and Electrical Communications, Faculty of Electronic \\ Engineering, Univ. of Menoufia32952, Menouf, Egypt. \\ m2alaa@hotmail.com \\ ${ }^{3}$ Dept. of Information Technology, Faculty of Computers and Information, \\ Univ. of Menoufia, 32511, ShebinElkom, Egypt. \\ mmadhoudeyahoo. com
}

\begin{abstract}
The contrast enhancement of medical images has an important role in diseases diagnostic, specially, cancer cases. Histogram equalization is considered as the most popular algorithm for contrast enhancement according to its effectiveness and simplicity. In this paper, we present a modified version of the Histogram Based Fast Enhancement Algorithm. This algorithm enhances the areas of interest with less complexity. It is applied only to CT head images and its idea based on treating with the soft tissues and ignoring other details in the image. The proposed modification make the algorithm is valid for most CT image types with enhanced results.
\end{abstract}

\section{KEYWORDS}

Contrast enhancement, Histogram equalization, Histogram Based Fast Enhancement Algorithm, CT image

\section{INTRODUCTION}

Diagnosing diseases using medical images becomes more popular using different types of imaging techniques. Computed tomography (CT) is considered as the best of them after developed in 1970's [1], especially in cancer detection [2]. Its idea depends on specializing gray level for every different organic tissue. Contrast of an image is defined as the ratio between the brightest and the darkest pixel intensities.

Histogram Equalization (HE) is considered as the most popular algorithm for contrast enhancement according to its effectiveness and simplicity. Its basic idea lies in mapping the gray

David C. Wyld et al. (Eds) : CST, ITCS, JSE, SIP, ARIA, DMS - 2014

pp. 261-269, 2014. (C) CS \& IT-CSCP 2014

DOI : $10.5121 /$ csit.2014.4124 
levels based on the probability distribution of the input gray levels. It flattens and stretches the dynamic range of the image's histogram, resulting in an overall contrast improvement.HE has been applied in various fields such as medical image processing and radar image processing [3, 4]. The two categories of histogram equalization are. Global histogram equalization, which is simple and fast, but its contrast-enhancement power is relatively low. Local histogram equalization, on the other hand, can effectively enhance contrast, but it requires more computations.

Global Histogram equalization is powerful in highlighting the borders and edges between different objects, but may reduce the local details within these objects [5] to overcome HE's problems. Ketcham and et al invented Local Histogram Equalization (LHE); the algorithm uses the histogram of a window of a predetermined size to determine the transformation of each pixel in the image. LHE succeeded in enhancing local details, but it depends on fixed size for windows where it may distort the boundaries between regions. It also demands high computational cost and sometimes causes over-enhancement in some portion of the image [6, 7].

There are many algorithms trying to preserve the brightness of the output image like BBHE (Brightness preserving Bi-Histogram Equalization) which separates the input image histogram into two parts based on the mean of the input image and then each part is equalized independently. There are many methods similar to BBHE like, DSIHE (Dualistic Sub-Image Histogram Equalization) where, it divides the histogram based on the median value. MDSIHE (Modified Dualistic Sub Image Histogram Equalization), A. Zadbuke made a modification on DSIHE and obtainedgood results [8]. MMBEBHE (Minimum Mean Brightness Error BiHistogram Equalization) provides maximal brightness preservation, but its resultsare foundnot good for the image with a lot details. To overcome these drawbacks, P. Jagatheeswari and et al proposed a modification to this method. They enhanced images by passing the enhanced ones through a median filter. The median filter is an effective method for the removal of impulse based noise on the images [9]. Recursive Mean-Separate Histogram Equalization (RMSHE) is also considered asan extension to BBHE. All these methods achieve good contrast but they have some problems in gray level variation [7].

The rest of the paper is organized as follows. in section 2, the idea of A Histogram-Based Fast Enhancement Algorithm will be introduced. Then, the problems were found in this algorithm and the suggested modification is presented in section 3. Experimental results using clinical data of CT images is discussed in section 4 to demonstrate the usefulness of the proposed method. Concluding remarks ispresented in section 5.

\section{A Histogram BASED FAST ENHANCEMENT ALgORITHM}

J. Yin and et al proposed an algorithm to enhance local interested areas in CT head images; they tried to improve the water-washed effect caused by the conventional histogram equalization algorithms as shown in Figure1. The algorithm succeeded in removing water-washed effect. There are some important features for this algorithm like the speed and the simplicity. Its idea depends on that, most CT head images occupy the gray level 0 so they try to deal with the soft tissues by enhancing the region by using full range of all possible gray levels to enhance it in the CT head images. They analyzed these images and found that more than half of the whole range of gray levels occupies 0 level, and all CT head images have three major peaks in their histograms. The left peak is formed by background pixels, the middle peak is usually formed by soft tissues in 
the CT head images, and the right peak is formed mostly by bone. For enhancement details, we need only the middle peak which formed by soft tissue [10].

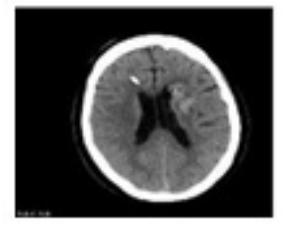

(a)

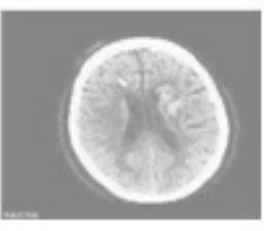

(b)



(c)

Figure 1. (a) an original CT head image (b) enhanced by conventional histogram equalization algorithm (c) Histogram-Based Fast Enhancement Algorithm.

\section{A MODIFIED HISTOGRAM BASED FAST ENHANCEMENT ALGORITHM}

The idea of the algorithm depends on the characteristics of CT head images. This makesthe algorithm is suitable for special type of images, so we tried to make a modification to this algorithm to be more appropriate for a wide range of CT images with enhanced results.The calculations of Histogram-Based Fast Enhancement Algorithm depends on a constant value $\mathrm{k}$ $(0<\mathrm{k}<0.4)$ to evaluate how many gray levels should be ignored. This means that $\mathrm{k}$ remains constant for all images regardlessof image characteristics, so we calculated the value of $\mathrm{k}$ to change with the gray levels of the picture.

First, we evaluated $\mathrm{k}$ as a ratio of the mean value of histogram values, which is considered as an importantfeature of the histogram then we recorded these results, and compared it with the Histogram-Based Fast Enhancement Algorithm; we found that there is a valuable enhancement in results. Thesteps of our proposed solution remained as in the Histogram-Based Fast Enhancement Algorithm, but the change will be occurred in determining k value as below.

$$
k=\text { ratio } * H_{\text {mean }}
$$

Where Hmean is the mean value of the histogram, which is the sum of the values divided by the number of values.Second, we performedanother modification by using $\mathrm{k}$ as a ratio of median value of the histogram and found that the results become better that because the value depends on the characteristic of image.

$$
k=\text { ratio } * H_{\text {median }}
$$

WhereHmedian is the median value of the histogram, it is the value which divides the values into two equal halves.At the last, we use the mode value as the most frequently occurring value in the histogram.

$$
k=\text { ratio } * H_{\text {mode }}
$$

We applied the modified algorithm to large varieties of CT images including head and lung images. To evaluate the effectiveness of the modification we use three widely-used metrics; PSNR (Peak Signal-to-Noise Ratio), AMBE (Absolute Mean Brightness Error), and the entropy, in addition to Inspection of Visual Quality. We will show briefly how to evaluate these metrics in the next section. 


\subsection{Peak Signal to Noise Ratio (PSNR)}

PSNR is the evaluation standard of the reconstructed image quality, and is an important measurement feature. PSNR is measured in decibels $(\mathrm{dB})$. If we suppose a reference image $f$ and a test image $t$, both of size $M \times N$, the PSNR between $f$ and $g$ is defined by.

$$
\operatorname{PSNR}(f, t)=\log _{10}(L-1)^{2} / \operatorname{MSE}(f, t)
$$

Where L is gray levels and MSE (Mean square error), is then defined as.

$$
\operatorname{MSE}(f, t)=\frac{1}{M N} \sum_{i=1}^{M} \sum_{j=1}^{N}\left(f_{i j}-t_{i j}\right)^{2}
$$

Note that the greater the PSNR, the better the output image quality.

\subsection{Absolute Mean Brightness Error (AMBE)}

It is the difference between original and enhanced image and is given as.

$$
\operatorname{AMBE}(X, Y)=|X M-Y M|
$$

Where $X M$ is the mean of the input image $X=\{X(i, j)\}$ and $Y M$ is the mean of the output image $\mathrm{Y}=\{\mathrm{Y}(\mathrm{i}, \mathrm{j})\}$.

We try to preserve the brightness of the image to keep the image details, so if we reduce the difference this preserve the brightness of the image.

\subsection{Entropy}

Entropy is a statistical measure of randomness that can be used to characterize the texture of the input image. It is a useful tool to measure the Richness of the details in the output image [11].

$$
\operatorname{Ent}[P]=\sum_{i=1}^{n}\left(P_{i} \log _{2}\left(P_{i}\right)\right)
$$

\subsection{Inspection of Visual Quality}

In addition to the quantitative evaluation of contrast enhancement using the PSNR and entropy values, it is also important to qualitatively assess the contrast enhancement. The major goal of the qualitative assessment is to judge if the output image is visually acceptable to human eyes and has a natural appearance [8]. 


\section{EXPERIMENTAL RESULTS}

To show the effect of the proposed modification, we apply it on different types of CT images. We use head images like the original algorithmin addition to the lung images to be validate for more image types.

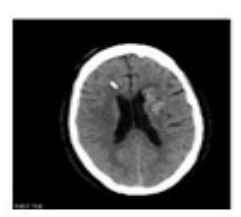

(a)

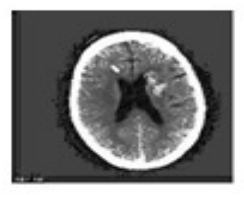

(d)

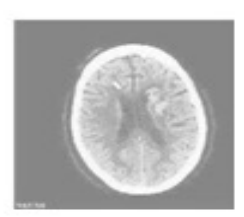

(b)

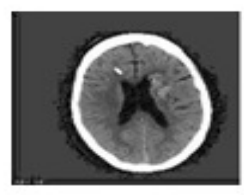

(e)

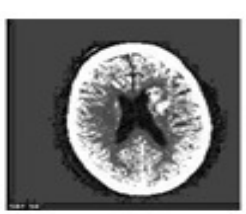

(c)

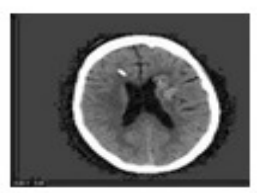

(f)

Figure 2. (a) Original CT head image (b) enhanced by conventional histogram equalization algorithm (c) enhanced by Histogram-Based Fast Enhancement Algorithm. (d) Modified Histogram-Based Fast Enhancement Algorithm using mean value (e) Modified Histogram-Based Fast Enhancement Algorithm using median value. (f) Modified Histogram-Based Fast Enhancement Algorithm using mode value

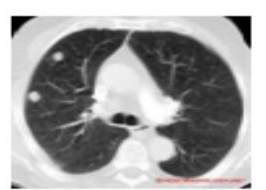

(a)

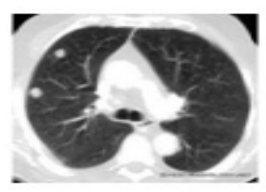

(d)

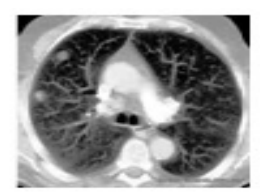

(b)

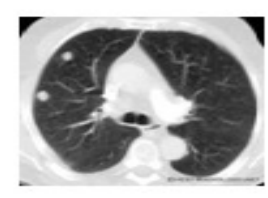

(e)

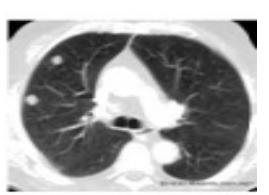

(c)

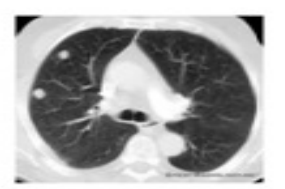

(f)

Figure 3. (a) Original CT lung image (b) enhanced by conventional histogram equalization algorithm (c) enhanced by Histogram-Based Fast Enhancement Algorithm. (d) Modified Histogram-Based Fast Enhancement Algorithm using mean value (e) Modified Histogram-Based Fast Enhancement Algorithm using median value (f) Modified Histogram-Based Fast Enhancement Algorithm using mode value 
Table 1. PSNR measurement

\begin{tabular}{|c|c|c|c|c|c|}
\hline \multirow[t]{2}{*}{ Image } & \multirow{2}{*}{$\begin{array}{l}\text { Conventional } \\
\text { Histogram } \\
\text { Equalization } \\
\text { Algorithm }\end{array}$} & \multirow{2}{*}{$\begin{array}{l}\text { Histogram- } \\
\text { Based Fast } \\
\text { Enhanceme } \\
\text { nt } \\
\text { Algorithm }\end{array}$} & \multicolumn{3}{|c|}{$\begin{array}{l}\text { Modified Histogram-Based Fast Enhancement } \\
\text { Algorithm }\end{array}$} \\
\hline & & & $\begin{array}{l}\text { Using } \\
\text { Mean }\end{array}$ & Mediang & fsing Mode \\
\hline CThead1 & 6.6589 & 12.1687 & 13.9531 & 14.6326 & 14.63262 \\
\hline CThead2 & 6.7181 & 12.3103 & 14.8156 & 14.81723 & 14.81723 \\
\hline CThead3 & 4.2788 & 9.0203 & 9.8088 & 11.22233 & 11.22233 \\
\hline CThead4 & 6.7181 & 12.3103 & 14.8156 & 14.81723 & 14.81723 \\
\hline CTlung1 & 17.9699 & 26.9840 & 28.6100 & 32.35675 & 34.2496 \\
\hline CTlung2 & 19.3186 & 30.1420 & 32.3924 & 41.8492 & 43.58417 \\
\hline CTlung3 & 8.839 & 13.8357 & 13.4644 & 14.50487 & 14.5052 \\
\hline CTlung4 & 15.3099 & 21.5727 & 26.8872 & 31.9475 & 35.1296 \\
\hline
\end{tabular}

As we mention before, the increase in the value of PSNR is considered as an enhancement in the algorithm. From Table 1 we find that there is an enhancement using the proposed modified algorithm.

Table 2. AMBE measurement.

\begin{tabular}{|c|c|c|c|c|c|}
\hline \multirow[b]{2}{*}{ Image } & \multirow{2}{*}{$\begin{array}{l}\text { Conventional } \\
\text { Histogram } \\
\text { Equalization } \\
\text { Algorithm }\end{array}$} & \multirow{2}{*}{$\begin{array}{l}\text { Histogram-Based } \\
\text { Fast } \\
\text { Enhancement } \\
\text { Algorithm }\end{array}$} & \multicolumn{3}{|c|}{$\begin{array}{l}\text { Modified Histogram-Based } \\
\text { Enhancement Algorithm }\end{array}$} \\
\hline & & & $\begin{array}{l}\text { Using } \\
\text { Mean }\end{array}$ & Jsing Median & Jsing Mode \\
\hline CThead1 & 111.8702 & 48.14349 & 38.0466 & 34.55872 & 34.55872 \\
\hline CThead2 & 97.365 & 41.37939 & 133.7495 & 30.06355 & 13.3852 \\
\hline CThead3 & 150.4411 & 78.69606 & 70.7215 & 57.66641 & 57.66641 \\
\hline CThead4 & 112.059 & 47.4835 & 33.5147 & 33.43602 & 33.43602 \\
\hline CTlung1 & 13.4576 & 4.9821 & 3.427556 & 1.969327 & 1.6462 \\
\hline CTlung2 & 15.1077 & 4.1489 & 3.2355 & 1.5521 & 1.369413 \\
\hline CTlung3 & 76.9961 & 41.85713 & 43.53475 & 35.29687 & 35.26233 \\
\hline CTlung4 & 13.977 & 11.785 & 5.95788 & 3.7327 & 2.9718 \\
\hline
\end{tabular}

Our Proposed algorithm is considered one of brightness persevered algorithm so we try to reduce the difference between the brightness of input and the result image. From Table 2, we can conclude that there is an enhancement in AMBE values using the proposed algorithm.

As we will see in Table 3, there is a small increase in the Entropy values especially using the median and the mode where we have found there is a great convergence between median and mode values. As for the Inspection of Visual Quality, as we see in Figure 2 and Figure 3 there are some details appeared in the proposed algorithm which help in diagnostic diseases more accurate. 
Table 3. Entropy measurement.

\begin{tabular}{|c|c|c|c|c|c|c|}
\hline \multirow{2}{*}{ Image } & \multirow[t]{2}{*}{$\begin{array}{l}\text { Original } \\
\text { Image }\end{array}$} & \multirow{2}{*}{$\begin{array}{c}\text { Conventional } \\
\text { Histogram } \\
\text { Equalizatio } \\
\mathbf{n} \\
\text { Algorithm }\end{array}$} & \multirow{2}{*}{$\begin{array}{l}\text { Histogram- } \\
\text { Based Fast } \\
\text { Enhancem } \\
\text { ent } \\
\text { Algorithm }\end{array}$} & \multicolumn{3}{|c|}{$\begin{array}{c}\text { Modified Histogram-Based } \\
\text { Enhancement Algorithm }\end{array}$} \\
\hline & & & & $\begin{array}{l}\text { Using } \\
\text { Mean }\end{array}$ & \begin{tabular}{|l} 
Jsing \\
Median
\end{tabular} & Jsing Mode \\
\hline CThead1 & 0.9991 & 3.3235 & 4.608886 & 4.912558 & 5.133528 & 5.13352 \\
\hline CThead2 & 0.8993 & 4.5394 & 5.144703 & 2.077121 & 5.391977 & 5.8384 \\
\hline CThead3 & 0.9169 & 2.3727 & 2.812813 & 2.9972 & 3.377863 & 3.37786 \\
\hline CThead4 & 0.9997 & 3.3564 & 4.4211 & 5.0363 & 5.058119 & 5.05811 \\
\hline CTlung1 & 0.0022 & 5.8899 & 6.9246 & 7.08508 & 7.102342 & 7.0458 \\
\hline CTlung2 & 0.0695 & 5.9528 & 6.8429 & 6.9279 & 7.1620 & 7.20219 \\
\hline CTlung3 & 0.1575 & 3.3206 & 3.517687 & 3.409321 & 4.483833 & 4.50024 \\
\hline CTlung4 & 0.2065 & 2.5454 & 6.4606 & 6.687 & 6.687 & 6.6064 \\
\hline
\end{tabular}

We can exclude some points from the previous results that the modified algorithm achieves greater values of PSNR, AMBE and entropy compared with Histogram-Based Fast Enhancement Algorithm. The first metric of PSNR; the propose algorithm have increased the values of PSNR; this means that less noise in the resulted image. The second metric is AMBE, it has been minimized and this means that it has preserved the brightness of the image. The third metric of entropy where it has increased; this means that more information can be extracted from the output image. We also performed statistical analysis for the results in Figure4, Figure5, and Figure6, where Figure4 shows the increment in PSNR values due to using the modification with mean, median and mode.Figure5 shows the enhancement in entropy values and Figure6 show the decrement of AMBE. There is a valuable improvement in the three parameters for the modification especially the mode where give the best results. We found that there is a range of ratio values that gives the best results for the three parameters and outside this range there arenot good results. This gives us the ability to control this ratio to obtain the best results.

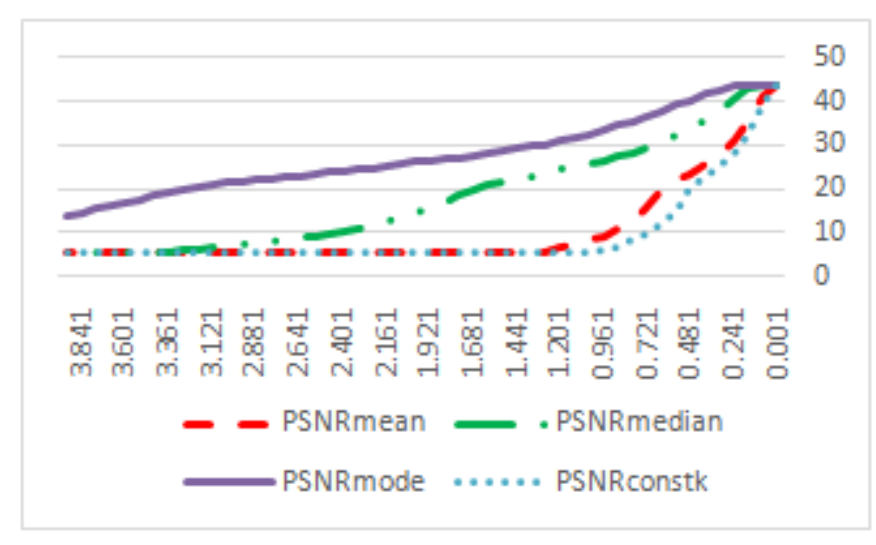

Figure4. The effect of modification on PSNR values 


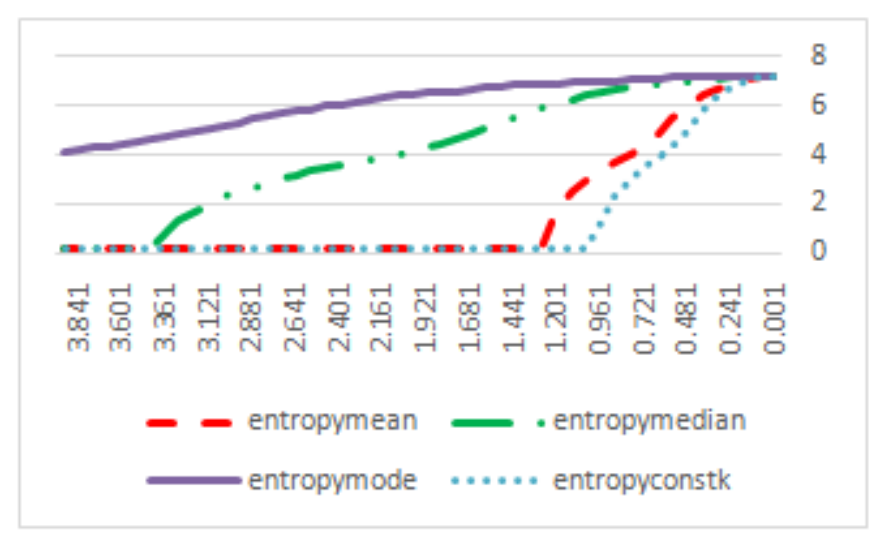

Figure5.The effect of modification on entropy values

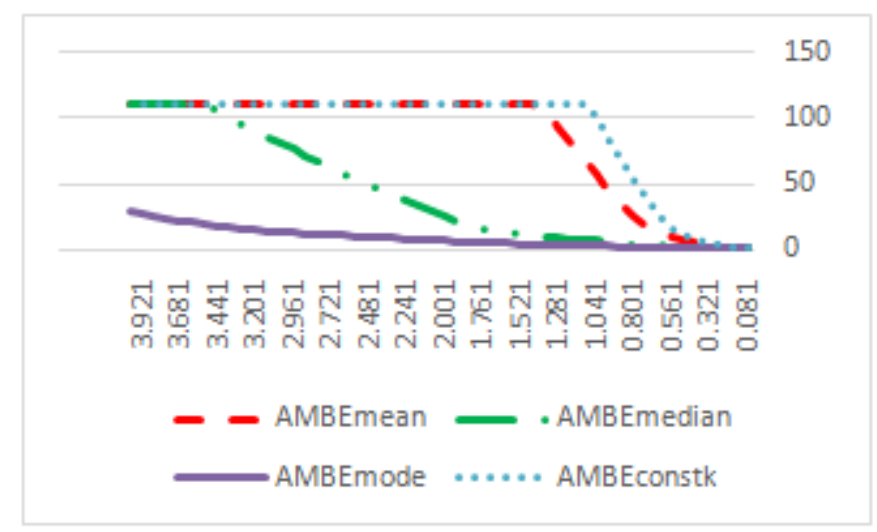

Figure6.The effect of modification on AMBE values

\section{CONCLUSION}

In this paper, we have presented a simple modificationof Histogram Based Fast Enhancement Algorithm. First, we have showed how it succeeded in removing water-washed effect. Then discuss the proposed modification which enhances the PSNR, AMBE and entropy parameters values to be more appropriate for a wide range of $\mathrm{CT}$ images.In addition to the enhancements occurred to the Histogram-Based Fast Enhancement Algorithm. There are some advantages of the algorithm compared to other algorithms. It still keeps the advantage of simplicity due to less complex calculations used in the algorithm. There is another advantage of this algorithm due to its idea of using global histogram and not based on local histogram. This decreases the used time for running.

\section{REFERENCES}

[1] S. Smith, (2002) "The Scientist and Engineer's Guide to Digital Signal Processing", Chapter 25: Special Imaging Techniques, Computed Tomography, Softcover,ISBN 0-7506-7444-X.

[2] Dr. West,"Lung Cancer Screening Saves Lives", http://cancergrace.org/lung/2010/11/04/lung-cancerscreening-saves-lives.

[3] Y. Kim, Feb.(1997) "Contrast enhancement using brightness preserving Bi-Histogram equalization", IEEE Trans. Consumer Electronics, vol. 43,no. 1, pp. 1-8. 
[4] R. Krutsch and D. Tenorio,(2011)"Histogram Equalization", Document Number: AN4318, Application Note Rev. 0.

[5] I. Jafar and H. Ying, (2007)" Multilevel Component-Based Histogram Equalization for Enhancing the Quality of Grayscale Images", IEEE EIT, pp. 563-568.

[6] R. Jonesand T. Tjahjadi, (1993)"A study and Modification of the Local Histogram Equalization Algorithm", pattern recognition, vol2, no. 9, pp 1373-1381.

[7] P. Shanmugavadivu, K. Balasubramania, (2010) "Image Inversion and Bi Level Histogram Equalization for Contrast Enhancement" International Journal of Computer Applications (0975 8887) Volume 1 - No. 15.

[8] A. Zadbuke,(2012)"Brightness Preserving Image Enhancement Using Modified Dualistic Sub Image Histogram Equalization", International Journal of Scientific \& Engineering Research, Volume 3, Issue 2, 1 ISSN 2229-5518.

[9] P. Jagatheeswari, S. Suresh Kumar, M. Rajaram, (2009)" Contrast Enhancement for Medical Images Based on Histogram Equalization Followed by Median Filter", the International Conference on ManMachine Systems (ICoMMS), MALAYSIA.

[10] J.Yin, X. Tian, Z. Tang, Y. Sun, (2006)"A Histogram-Based Fast Enhancement Algorithm for CT Head Images", Intl. Conf. on Biomedical and Pharmaceutical Engineering (ICB PE).

[11] "Image entropy",http://www.esrf.eu/computing/Forum/imgCIF/PAPER/entropy. 